\title{
Adherence to the pro-inflammatory diet in relation to prevalence of irritable bowel syndrome
}

\author{
Asma Salari-Moghaddam ${ }^{1,2}$, Ammar Hassanzadeh Keshteli ${ }^{3,4}$, Ahmad Esmaillzadeh ${ }^{2,5,6^{*}}$ and Peyman Adibi ${ }^{4}$
}

\begin{abstract}
Objective: There is no prior study that examined the association between nutrient-based dietary inflammatory index (DII) and odds of Irritable Bowel Syndrome (IBS). We examined the association between DII score and odds of IBS and its severity among Iranian adults.

Methods: In this cross-sectional study, dietary intakes of 3363 Iranian adults were examined using a validated Dishbased 106-item Semi-quantitative Food Frequency Questionnaire (DS-FFQ). DIl was calculated based on dietary intakes derived from DS-FFQ. IBS was assessed using a modified Persian version of Rome III questionnaire.

Results: After adjustment for potential confounders, we found that participants in the highest quintile of DII score had greater chance for IBS compared with those in the lowest quintile (OR: 1.36; 95\% Cl: 1.03-1.80). By gender, we found a significant association between DII score and IBS among women (OR: 1.41; 95\% Cl: 1.00-2.00). By BMI status, overweight or obese (BMI $\left.\geq 25 \mathrm{~kg} / \mathrm{m}^{2}\right)$ individuals in top quintile of DII score had greater odds for IBS than those in the bottom quintile (OR: 1.64; $95 \%$ Cl: 1.07-2.53). No significant association was observed between a proinflammatory diet and severity of IBS symptoms.
\end{abstract}

Conclusions: Consumption of a pro-inflammatory diet was associated with increased odds of IBS, in particular among women and those with $\mathrm{BMl} \geq 25 \mathrm{~kg} / \mathrm{m}^{2}$.

Keywords: Dietary inflammatory index, DII, Inflammation, Irritable bowel syndrome, IBS

\section{Introduction}

Irritable Bowel Syndrome (IBS) is one of the most common functional gastrointestinal disorders [1] which presents by abdominal pain and changes in bowel habits [2]. This condition affects $11 \%$ of worldwide population [3]. The high prevalence of IBS is also reported from Iran; such that, it has been shown that 1.1 to $25 \%$ of Iranian adults are affected by this condition [4].

The etiology of IBS is not well recognized; however, several factors including genetic susceptibility, female gender, family history and dietary factors might contribute

\footnotetext{
* Correspondence: a-esmaillzadeh@tums.ac.ir

${ }^{2}$ Department of Community Nutrition, School of Nutritional Sciences and Dietetics, Tehran University of Medical Sciences, P.O. Box 14155-6117, Tehran, Iran

${ }^{5}$ Obesity and Eating Habits Research Center, Endocrinology and Metabolism Molecular-Cellular Sciences Institute, Tehran University of Medical Sciences, Tehran, Iran

Full list of author information is available at the end of the article
}

to this condition [5-8]. Inflammation has been postulated to play a key role in pathology of IBS. Low grade inflammation contributes to the GI motor dysfunction and abdominal symptoms in patients with GI disorders. Low grade inflammation in the mucosal compartment of the gut can alter function in the underlying neuromuscular tissues from animal studies [9]. Individuals with IBS have been shown to have high levels of low-grade systemic inflammation [10]. Therefore, potential factors that increase systemic inflammation might be involved in the incidence and exacerbation of IBS symptoms. Among others, dietary factors are the most important one due to their unavoidable universal exposure to all people [11]. Dietary factors stimulating inflammatory process might be involved in the IBS pathology. To assess the inflammatory potential of the diet, recently Dietary Inflammatory Index (DII) has been constructed, which categorizes individual's diet on a continuum from maximally anti-inflammatory to

(C) The Author(s). 2019 Open Access This article is distributed under the terms of the Creative Commons Attribution 4.0 International License (http://creativecommons.org/licenses/by/4.0/), which permits unrestricted use, distribution, and 
maximally pro-inflammatory [12]. The association between DII and inflammatory markers has been reported in earlier studies [13, 14]. Chronic proinflammatory conditions including obesity, metabolic syndrome and cardiovascular diseases have been extensively linked with DII [15-17]. However, no earlier study has assessed the association between DII and IBS. In a case-control study on patients with inflammatory bowel disease, consumption of pro-inflammatory diet has been associated with greater risk of inflammatory bowel disease [18]. In addition, adherence to western dietary pattern, which is mostly a pro-inflammatory diet, was prospectively associated with increased risk of IBS [19]. Consumption of fast food dietary pattern was also linked with greater odds of IBS [20].

The inflammatory potential of the diet can be examined by considering the anti- and pro-inflammatory properties of nutrients and foods. In a previous study, we developed an empirically-derived food-based dietary inflammatory index (FDII) and examined its association with IBS [21]. In that study, we found that consumption of a pro-inflammatory diet was associated with increased risk for IBS. However, nutrient-based DII might be differently associated with IBS and its severity because of the interactions among nutrients and their synergistic effects on each other in the gastrointestinal lumen. To our knowledge, there is no study on the association between nutrient-based DII and odds of IBS. In addition, most studies on the association between diet and IBS have focused on dietary components that alleviate IBS symptoms. Moreover, previous studies on the association of DII and chronic diseases have mostly focused on metabolic abnormalities rather than non-metabolic diseases. Finding the association of DII with non-metabolic conditions, including IBS, may help expanding the application of this index in dietary recommendations. This study was done to examine the association between DII score and IBS in a large sample of Iranian adults.

\section{Materials and methods Participants}

This cross-sectional study was conducted within the framework of the Study on the Epidemiology of Psychological, Alimentary Health and Nutrition (SEPAHAN) project, a cross-sectional study that investigated the prevalence of functional gastrointestinal disorders (FGIDs) and their relationship with lifestyle factors. Details about SEPAHAN project have been published elsewhere [22]. Inclusion criteria for this study were as follow: Iranian general adults (aged $18-55$ years) working in 50 different healthcare centers affiliated to Isfahan University of Medical Sciences (IUMS) across Isfahan province. In this project, data were collected in 2 main phases between April 2010 and May 2010. To collect information about anthropometric indices, demographic and lifestyle factors, including dietary intakes and physical activity, self-administered questionnaires distributed among 10,087 subjects in the first phase, and 8691 participants returned the completed questionnaires (response rate: $86.16 \%$ ). In the second phase, data regarding gastrointestinal health were collected (response rate: 64.6\%). Finally, we were able to match 4763 questionnaires in the second phase with their corresponding questionnaires in the first phase. In the current study, we excluded subjects who had total daily energy intakes outside the range of $800-4200 \mathrm{kcal} / \mathrm{d}$ as well as those that had missing data on any relevant variable. Therefore, data from 3363 subjects, for whom complete information about both dietary intakes and IBS were available, were included in the current analysis. All participants provided written inform consent forms. The study protocol was ethically approved by the Regional Bioethics Committee of Isfahan University of Medical Sciences.

\section{Dietary intakes assessment}

Dietary data were collected using a Willett-format dish-based 106-item semi-quantitative food frequency questionnaire (DS-FFQ) which was designed and validated specifically for Iranian adults. Detailed information about the design, foods included, and the validity of this questionnaire has been published elsewhere [23]. Briefly, the questionnaire contained five categories of foods and dishes: 1) mixed dishes (cooked or canned, 29 items); 2) grains (different types of bread, cakes, biscuits and potato, 10 items); 3) dairy products (dairies, butter, and cream, 9 items); 4) fruits and vegetables (22 items); and 5) miscellaneous food items and beverages (including sweets, fast foods, nuts, desserts and beverages, 36 items). For each food item, a commonly consumed portion size was defined. Participants were asked to report their dietary intakes of foods and mixed dishes based on nine multiple choice frequency response categories varying from "never or less than once a month" to " 12 or more times per day". The frequency response categories for the food list varied from six to nine choices. For foods consumed infrequently, we omitted the high-frequency categories, while for common foods with a high consumption, the number of multiple choice categories increased. For instance, the frequency response for tuna consumption included six categories, as follows: never or less than once/month, 1-3 times/month, 1 time per week, 2-4 times/week, 5-6 times/week, 1-2 times/day; and for tea consumption that is highly prevalent among Iranians, the frequency response included nine categories, as follows: never or less than 1 cup/month, 1-3 cups/month, 1-3 cups/week, 4-6 cups/week, 1 cup/day, 2-4 cups/day, 5-7 cups/day, 8- 
$11 \mathrm{cups} /$ day, $\geq 12 \mathrm{cups} /$ day). Finally, to convert the food items into grams, we computed the amount of each portion size based on the booklet of "household measures" and then computed the amount of intake by considering the frequency of consumption of each food item. The validity of DS-FFQ was examined in a subgroup of 200 randomly selected participants of SEPAHAN project. All participants in the validation study completed the DS-FFQ at study baseline and 6 months later. During this validation study, participants provided three detailed dietary records that were used as gold standard. As shown in earlier studies [23], it seems that this questionnaire provides reasonably valid measures of long-term dietary intakes.

\section{Assessment of dietary inflammatory index}

Dietary data derived from DS-FFQ were used to calculate DII scores for all subjects. Earlier studies reported the development [12] and construct validation of the DII $[24,25]$. Shivappa et al. [12] found that a total of 45 specific foods and nutrients were associated with one or more of the inflammatory [Interleukin-1 $\beta$ (IL-1 $\beta)$, Interleukin-6 (IL-6), Tumor Necrosis Factor- $\alpha$ (TNF- $\alpha$ ) or CRP] or anti-inflammatory biomarkers [Interleukin-4 (IL-4) and Interleukin-10 (IL-10)]. Then, they scored the inflammatory potential for each food parameter according to whether it increased inflammatory or decreased anti-inflammatory factors $(+1)$, or it decreased inflammatory or increased anti-inflammatory factors $(-1)$, or had no effect (0) on inflammatory or anti-inflammatory biomarkers. They calculated world mean and standard deviation for each of the 45 food parameters based on 11 data sets from 11 countries in different parts of the world. Due to lack of consumption of some foods in Iranian dietary culture as well as missing some items (like polyphenols) in our nutrient database, in the current study we calculated DII score based on 29 food parameters (rather than 45). The food parameters we used in the current study were as follow: pro-inflammatory parameters included energy, carbohydrate, fat, protein, cholesterol, saturated fat, trans fat, vitamin B12 and iron and anti-inflammatory parameters included mono-unsaturated fatty acids (MUFA), poly unsaturated fatty acids (PUFA), fiber, vitamin B6, folic acid, niacin, riboflavin, thiamin, vitamin $A$, vitamin $C$, vitamin $D$, vitamin $E, \beta$-carotene, caffeine, pepper, onion, tea, zinc, selenium, and magnesium. First, we calculated energy-adjusted amounts of these nutrients using residual method. Then, to calculate DII score for each participant, we calculated the $z$ score for a given food parameter by subtracting the "standard global mean" from the amount consumed by each subject and dividing this value by the "global standard deviation". Global means and standard deviations were obtained from the study of Shivappa et al. [12] We converted this value to a centered percentile score in order to reduce skewness, as earlier studies did [12]. For each participant, this score was then multiplied by the respective food parameter effect score derived from the study of Shivappa et al. [12]. Then, we calculated overall DII score for each participant by summing up all foods' DII score. A higher DII score (more positive) indicates a more inflammatory diet and a lower DII score (more negative) indicates a less inflammatory diet.

\section{Assessment of IBS}

A modified Persian version of the Rome III questionnaire, as part of the main comprehensive questionnaire, was used for assessment of IBS. During the face validation of the questionnaire, we found that most participants were unable to distinguish between the descriptors used in the original Rome III questionnaire (never, less than 1 day a month, 1 day a month, 2-3 days a month, 1 day a week, more than 1 day a week, every day). We, therefore, modified the rating scales to consist of only four descriptors (i.e., never or rarely, sometimes, often, and always). We also decided to ask about the presence of each symptom in the past 3 months instead of questioning patients about the beginning of each symptom in more than 6 months prior to the evaluation, which already exists in original ROME III questionnaire. IBS was defined according to ROME III criteria as having recurrent abdominal pain or discomfort, at least sometimes, in the last 3 months associated with two or more of these criteria: improvement with defecation at least sometimes and onset associated with change in frequency or form (appearance) of stool, at least sometimes. IBS with constipation was defined as having IBS and both of the following criteria: (i) hard or lumpy stools at least sometimes and (ii) lack of loose, mushy or watery stools. IBS with diarrhea was defined as having IBS and both of the following criteria: (i) lack of hard or lumpy stools and (ii) loose, mushy or watery stools at least sometimes. Mixed IBS was defined as having IBS and both of the following criteria: (i) hard or lumpy stools at least sometimes and (ii) loose, mushy or watery stools at least sometimes. The severity of IBS was examined by asking participants on the severity of their abdominal pain in the last 3 months. They were able to choose one of these responses: mild/moderate/severe and very severe.

\section{Assessment of other variables}

Required information on other variables including age, sex, marital status, smoking status, medication use and disease history (diabetes and colitis) was obtained from demographic and medical history questionnaires. Physical activity was assessed using the General Practice Physical Activity Questionnaire (GPPAQ) [26], and 
participants were classified into two categories: physically active $(\geq 1 \mathrm{~h} /$ week $)$ and physically inactive $(<1 \mathrm{~h} /$ week). Although this level of activity might seem low, but earlier publications have revealed that even $1 \mathrm{~h}$ per week of walking can reduce the risk of chronic conditions [27]. Data on diet-related practices including meal regularity (often or always/never or occasionally), chewing efficiency (a lot/not a lot) and intra-meal fluid intake $(<3$ glasses/ $\geq 3$ glasses $)$ were also assessed through the use of a pretested questionnaire. Dental status was also examined and subjects were categorized as "having all teeth", "lost 1-5 teeth" and "lost $>5$ teeth". Anthropometric measures including weight, height, and waist circumference were assessed using a self-administered questionnaire. The validity of self-reported values of weight, height, and waist circumferences (WC) was examined in a pilot study on 200 participants from the same population. In the validation study, self-reported values of anthropometric indices were compared with actually measured values. The correlation coefficients for self-reported weight, height, and WC versus corresponding measured values were $0.95(P<0.001), 0.83(P<$ $0.001)$, and $0.60(P<0.001)$, respectively. Body Mass Index (BMI) was calculated by dividing weight $(\mathrm{kg})$ to height $\left(\mathrm{m}^{2}\right)$. The correlation coefficient for computed BMI from self-reported values, and the one from measured values was $0.70(P<0.001)$.

\section{Statistical methods}

We classified participants based on quintile cut-off points of DII score. General characteristics of study participants across quintiles of DII score were presented as means \pm SDs for continuous variables and percentages for categorical variables. To examine the differences across quintiles, we used ANOVA for continuous variables and chi-square test for categorical variables. Energy-adjusted dietary intakes of study participants across quintiles of DII score were compared by using analysis of covariance (ANCOVA). We used binary logistic regression to estimate ORs and 95\% CIs for the presence of IBS and its subtypes across quintiles of DII score in crude and multivariableadjusted model. The trend of ORs across quintiles of DII score was determined by considering quintiles of DII score as ordinal variables in the logistic regression analysis. We also used multivariable ordinal logistic regression to estimate ORs and 95\% CIs for assessing IBS severity (mild/moderate/ severe/very severe) across quintiles of DII score in crude and multivariable-adjusted model. In these analyses, sex (male/female), smoking status (non-smoker/former smokers and current smokers), physical activity $(<1 \mathrm{~h} /$ week/ $/ 1 \mathrm{~h} /$ week), medication use (yes/no), self-reported diabetes (yes/ no) and colitis (yes/no), meal regularity (often or always/ never or occasionally), chewing sufficiency (a lot/not a lot), intra-meal fluid consumption ( $<3$ glasses $/ \geq 3$ glasses), and dental status (have all teeth/lost 1-5 teeth/lost $>5$ teeth) were adjusted for in the multivariable model. All statistical analyses were done using the Statistical Package for Social Sciences (version 20; SPSS Inc.). $P<0.05$ was considered as statistically significant.

\section{Results}

In the current study, the DII score ranged from -4.49 to +5.39 . Median overall DII score across increasing quintiles were $-2.05,-0.90,-0.02,+0.86$ and +2.06 , respectively. Overall, $22.2 \%$ of study participants had IBS $(n=748)$. General characteristics of study subjects are presented in Table 1. Compared with those in the lowest quintile, participants in the highest quintile of DII score were younger, less likely to be females, overweight, physically active and to have disease history of diabetes and colitis and more likely to have psychological distress. No significant differences were observed in terms of other variables across quintiles of DII score.

A greater DII score was significantly associated with higher intakes of energy, carbohydrates, saturated fats, trans fats, niacin, thiamin and caffeine and lower intakes of fats, proteins, dietary fiber, cholesterol, MUFA, PUFA, vitamin B12, vitamin B6, folic acid, riboflavin, vitamin A, vitamin $C$, vitamin $D$, vitamin $E, \beta$-carotene, pepper, onion, tea, zinc and magnesium (average consumption of these parameters has been published earlier) [28].

In the crude model, participants in the highest quintile of DII score had greater chance for IBS (OR: 1.34; 95\% CI: 1.04-1.73) compared with those in the lowest quintile (Fig. 1a). The association remained significant even after adjustment for potential confounders (OR: 1.36; 95\% CI: 1.03-1.80) (Fig. 1b).

Gender- and BMI-stratified analysis on the association of DII score and prevalent IBS are provided in Table 2 . When the analyses were done separately by gender, we found no significant association between DII score and IBS in men; however, among women, we observed a significant association between DII score and IBS in crude (OR: 1.54; 95\% CI: 1.12-2.13) and multivariable-adjusted model; such that those in the highest quintile of DII score were $41 \%$ more likely to have IBS than those in the lowest quintile after controlling for potential confounders (OR: 1.41; 95\% CI: 1.00-2.00). By BMI status, overweight and obese $\left(\mathrm{BMI} \geq 25 \mathrm{~kg} / \mathrm{m}^{2}\right)$ individuals in top quintile of DII score had greater odds for IBS than those in bottom quintile (OR: 1.64; 95\% CI: 1.07-2.53). However, we failed to find any significant association between DII score and IBS in normal weight (BMI $<25 \mathrm{~kg} /$ $\mathrm{m}^{2}$ ) individuals either before (OR: 1.37; 95\% CI: 0.951.96) or after controlling for potential confounders (OR: 1.22; 95\% CI: 0.83-1.80).

Crude and multivariable-adjusted ORs and 95\% CIs for IBS severity across quintiles of DII score are 
Table 1 General characteristics of study participants across quintiles of DIl score ${ }^{a}$

\begin{tabular}{|c|c|c|c|c|}
\hline \\
\hline \multicolumn{5}{|l|}{$\frac{\text { Quintiles of DII score }}{\text { Variables }}$} \\
\hline Dll range & -4.49 to -1.41 & -0.47 to +0.44 & +1.38 to +5.39 & \\
\hline Subjects, n & 672 & 672 & 672 & \\
\hline Age, y & $37.7 \pm 7.9$ & $36.8 \pm 7.7$ & $34.9 \pm 7.6$ & $<0.001$ \\
\hline $\mathrm{BMI}, \mathrm{kg} / \mathrm{m}^{2}$ & $25.4 \pm 3.9$ & $25.04 \pm 3.7$ & $24.2 \pm 3.6$ & $<0.001$ \\
\hline Psychological distress & $1.74 \pm 2.38$ & $1.93 \pm 2.65$ & $2.43 \pm 2.91$ & $<0.001$ \\
\hline Female, \% & 61.3 & 57.7 & 52.1 & 0.005 \\
\hline Married, \% & 81.7 & 83.4 & 79.9 & 0.62 \\
\hline Physically active, \% & 39.9 & 31.8 & 30.1 & $<0.001$ \\
\hline Current smokers, \% & 3.7 & 3.2 & 3.8 & 0.51 \\
\hline Disease history, \% & 5.8 & 4.3 & 4.8 & 0.04 \\
\hline Regular meal pattern, \% & & & & 0.18 \\
\hline Often or always & 62.8 & 62 & 57.3 & \\
\hline Never or occasionally & 37.2 & 38 & 42.7 & \\
\hline Chewing sufficiency, \% & & & & 0.36 \\
\hline A lot & 14.3 & 14.2 & 11 & \\
\hline Not a lot & 85.7 & 85.8 & 89 & \\
\hline Fluid consumption, $\%$ & & & & 0.72 \\
\hline$<3$ glasses & 97.2 & 96.1 & 96.5 & \\
\hline$\geq 3$ glasses & 2.8 & 3.9 & 3.5 & \\
\hline Tooth loss, \% & & & & 0.11 \\
\hline Have all & 32.6 & 30.6 & 37.4 & \\
\hline Lost $1-5$ teeth & 58.1 & 62.5 & 55.1 & \\
\hline Lost $>5$ teeth & 9.3 & 6.9 & 7.5 & \\
\hline
\end{tabular}

${ }^{a}$ Data are mean \pm standard deviation (SD)

${ }^{\mathrm{b}}$ Obtained from ANOVA or chi-square test, where appropriate

presented in Table 3. No significant association was observed between DII score and IBS severity in crude (OR: 1.23; 95\% CI: 0.77-1.96) or multivariable-adjusted model (OR: 1.08; 95\% CI: 0.65-1.80) in subjects with IBS. This was also the case when we analyzed data separately by gender or BMI status.

Crude and multivariable-adjusted ORs and 95\% CIs for IBS subtypes across quintiles of DII score are presented in Table 4. After controlling for potential confounders, we found that those with the greatest DII score had higher odds of IBS-M than those with the lowest DII score (OR: 1.90; 95\% CI: 1.00-3.59). However; after adjustment for potential confounders, this association became non-significant (OR: 1.65; 95\% CI: 0.843.23). No other overall association was seen between DII score and other types of IBS.

\section{Discussion}

In this cross-sectional study, we found that adherence to a pro-inflammatory diet was associated with increased odds of IBS in the whole population as well as in women, but not in men. There was a significant association between DII score and IBS in overweight and obese (BMI $\left.\geq 25 \mathrm{~kg} / \mathrm{m}^{2}\right)$ subjects; however, the association was not significant in normal weight (BMI $<25 \mathrm{~kg}$ / $\mathrm{m}^{2}$ ) participants. No significant association was observed between adherence to a pro-inflammatory diet and odds of IBS severity.

Irritable bowel syndrome is one of the most common gastrointestinal disorders [1]. Preventive strategies including lifestyle modifications are of great importance in this regard. Diet and inflammation have been proposed to play an important role in the etiology of IBS. We found a positive significant association between inflammatory potential of the diet and odds of IBS. To our knowledge, this is the first study that examined the association between a pro-inflammatory diet and IBS. It is well established that IBS is an inflammatory condition [9]. Therefore, it is expected that the pro-inflammatory potential of the whole diet might be associated with IBS. 
A

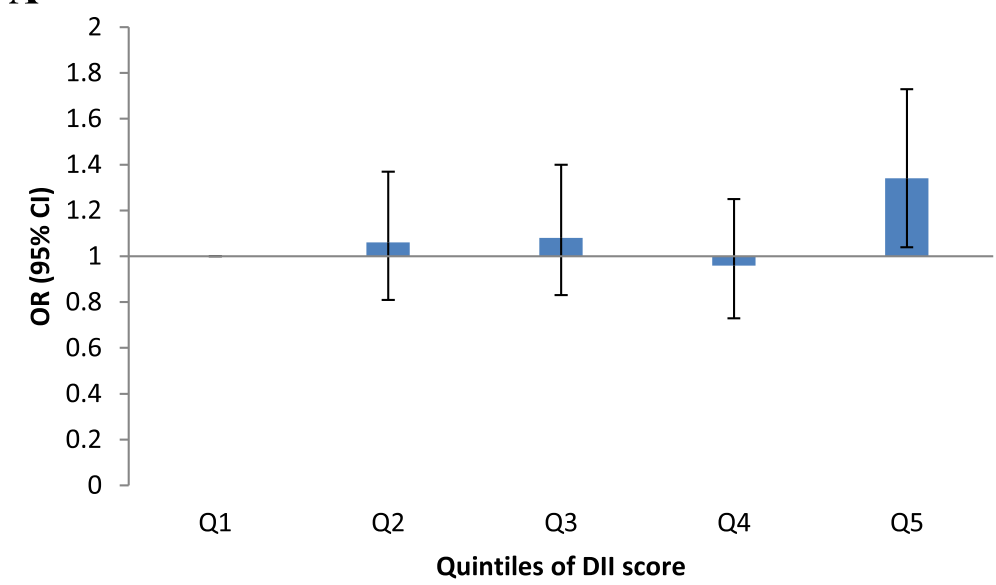

B

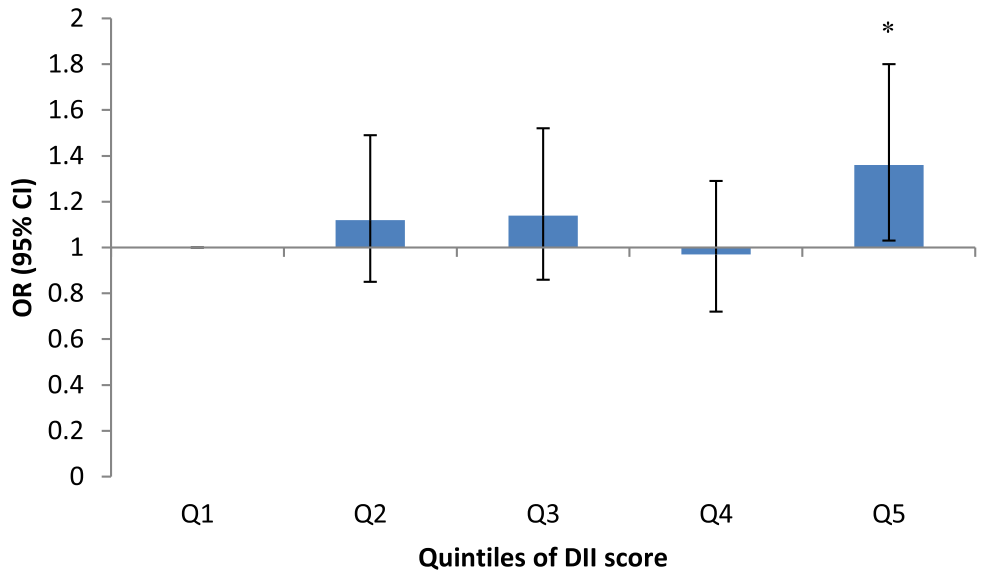

Fig. 1 a The association between DII score and IBS in whole population (crude model). $\mathbf{b}$ The association between DII score and IBS in whole population (multivariable-adjusted model); ${ }^{*} \mathrm{P}<0.001$ compared with the first quintile

It has been reported that IBS patients have lower intakes of fruits, vegetables and dairy products [29], all with anti-inflammatory properties. There is no previous study that examined the association of DII score and IBS; however, the association of this index with other proinflammatory conditions has been examined. In a casecontrol study, the investigators reported a significant association between DII score and ulcerative colitis [18]. The association of DII with diabetes, metabolic syndrome and obesity has also been observed in earlier studies $[15,16]$. These findings suggest that DII may elucidate the role of diet in the development of chronic diseases through inflammatory process.

We observed a gender difference in the association of a pro-inflammatory diet and IBS. Previous studies showed a higher prevalence of IBS among women than men [30]. These findings suggest a role for sex hormones in the etiology of IBS. Sex hormones may modulate IBS onset and exacerbation. In addition, slow GI transit, delayed gastric empty, and reduced colonic transit time among women than men might be mediated, at least in part, by sex hormones [31]. On the other hand, stress, a major contributing factor to IBS, is highly prevalent among women than in men [32]. Given the elevated levels of inflammation in stressed subjects and the probable interaction of diet with these situations, one might explain the gender difference in the association of pro-inflammatory potential of the diet and IBS.

We found a significant direct association between a pro-inflammatory diet and IBS among overweight and obese participants, but not in normal-weight subjects. This observation was in line with earlier studies that showed a higher prevalence of IBS in overweight and obese individuals compared with normal-weight subjects. A cross-sectional study that examined the association between gastrointestinal symptoms and BMI, reported a significant association between obesity and IBS symptoms [33]. Another study reached the same 
Table 2 Gender- and BMI-stratified odds ratios and 95\% Cls for IBS across quintiles of DII score ${ }^{a}$

\begin{tabular}{|c|c|c|c|c|}
\hline \multicolumn{5}{|l|}{ Quintiles of DII score } \\
\hline & $\mathrm{Q}_{1}$ & $\mathrm{Q}_{3}$ & $\mathrm{Q}_{5}$ & \\
\hline Median DII & -2.05 & -0.02 & +2.06 & $P$-trend \\
\hline \multicolumn{5}{|l|}{ Male } \\
\hline Crude & 1.00 & $1.35(0.87-2.08)$ & $1.22(0.80-1.88)$ & 0.46 \\
\hline Multivariable-adjusted $^{b}$ & 1.00 & $1.53(0.94-2.48)$ & $1.33(0.82-2.16)$ & 0.39 \\
\hline \multicolumn{5}{|l|}{ Female } \\
\hline Crude & 1.00 & $0.96(0.69-1.34)$ & $1.54(1.12-2.13)$ & 0.04 \\
\hline Multivariable-adjusted $^{\mathrm{b}}$ & 1.00 & $0.99(0.70-1.41)$ & $1.41(1.00-2.00)$ & 0.22 \\
\hline \multicolumn{5}{|l|}{$\mathrm{BMI}<25\left(\mathrm{~kg} / \mathrm{m}^{2}\right)$} \\
\hline Crude & 1.00 & $1.08(0.73-1.59)$ & $1.37(0.95-1.96)$ & 0.08 \\
\hline Multivariable-adjusted $^{c}$ & 1.00 & $1.06(0.70-1.59)$ & $1.22(0.83-1.80)$ & 0.45 \\
\hline \multicolumn{5}{|l|}{$\mathrm{BMI} \geq 25\left(\mathrm{~kg} / \mathrm{m}^{2}\right)$} \\
\hline Crude & 1.00 & $1.15(0.80-1.66)$ & $1.41(0.96-2.07)$ & 0.27 \\
\hline Multivariable-adjusted $^{c}$ & 1.00 & $1.32(0.88-1.98)$ & $1.64(1.07-2.53)$ & 0.09 \\
\hline
\end{tabular}

Table 3 Odds ratios and 95\% Cls for IBS severity across quintiles of Dll score ${ }^{a}$

\begin{tabular}{|c|c|c|c|}
\hline Median DII & $\begin{array}{l}Q_{1} \\
-2.05\end{array}$ & $\begin{array}{l}\mathrm{Q}_{3} \\
-0.02\end{array}$ & $\begin{array}{l}Q_{5} \\
+2.06\end{array}$ \\
\hline \multicolumn{4}{|l|}{ Whole population } \\
\hline Crude & 1.00 & $0.97(0.59-1.60)$ & $1.23(0.77-1.96)$ \\
\hline Multivariable-adjusted $^{b}$ & 1.00 & $0.90(0.53-1.55)$ & $1.08(0.65-1.80)$ \\
\hline \multicolumn{4}{|l|}{ Male } \\
\hline Crude & 1.00 & $0.67(0.27-1.66)$ & $1.34(0.59-3.04)$ \\
\hline Multivariable-adjusted $^{c}$ & 1.00 & $0.57(0.19-1.66)$ & $1.11(0.42-2.91)$ \\
\hline \multicolumn{4}{|l|}{ Female } \\
\hline Crude & 1.00 & $1.18(0.64-2.16)$ & $1.18(0.67-2.08)$ \\
\hline Multivariable-adjusted $^{c}$ & 1.00 & $1.11(0.59-2.12)$ & $1.07(0.58-1.97)$ \\
\hline \multicolumn{4}{|l|}{$\mathrm{BMl}<25\left(\mathrm{~kg} / \mathrm{m}^{2}\right)$} \\
\hline Crude & 1.00 & $0.70(0.33-1.47)$ & $0.81(0.41-1.60)$ \\
\hline Multivariable-adjusted $^{b}$ & 1.00 & $0.73(0.33-1.61)$ & $0.79(0.38-1.64)$ \\
\hline \multicolumn{4}{|l|}{$\mathrm{BMI} \geq 25\left(\mathrm{~kg} / \mathrm{m}^{2}\right)$} \\
\hline Crude & 1.00 & $1.32(0.64-2.70)$ & $1.70(0.85-3.38)$ \\
\hline Multivariable-adjusted $^{\mathrm{b}}$ & 1.00 & $1.12(0.50-2.51)$ & $1.52(0.70-3.32)$ \\
\hline
\end{tabular}

${ }^{\mathrm{a}}$ Values are OR $(95 \% \mathrm{Cls})$. IBS was defined as having recurrent abdominal pain or discomfort at least sometimes in the last 3 months associated with two or more of these criteria: improvement with defecation at least sometimes and onset associated with change in frequency or form (appearance) of stool at least sometimes

${ }^{\mathrm{b}}$ Adjusted for sex, physical activity, smoking status, medication use, disease history (diabetes, colitis), psychological distress, regular meal pattern, chewing sufficiency, fluid consumption and dental status

'Adjusted for physical activity, smoking status, medication use, disease history (diabetes, colitis), psychological distress, regular meal pattern, chewing sufficiency, fluid consumption and dental status

findings [34]. It is well known that overweight and obesity are associated with elevated levels of circulating inflammation [35]. High prevalence of IBS in obese subjects might also be explained by inflammation. Given the inflammatory nature of obesity and IBS, consumption of a pro-inflammatory diet might exacerbate these conditions.

We observed no significant association between a proinflammatory diet and IBS severity in the whole population as well as among overweight and obese subjects. Previous studies have shown a low-grade intestinal inflammation in IBS patients and consequent increased intestinal permeability [36]. A cross-sectional study showed higher prevalence of functional bowel symptoms in patients with morbid obesity [37]. Another crosssectional study in Sweden reported greater severity of symptoms in overweight and obese subjects with IBS compared with normal weight subjects with IBS [38]. Such association has been shown in other studies as well $[39,40]$. It has been shown that elevated inflammation would aggravate the IBS symptoms. Therefore, given the nature of irritable bowel syndrome, association between adherence to a pro-inflammatory diet and odds of IBS severity is expected. Lack of finding a significant association in the current study might be attributed to the assessment of IBS severity by only a question. It seems that accurate assessment of IBS severity needs further investigation and the severity of abdominal pain only, 
Table 4 Odds ratios and 95\% Cls for IBS subtypes across quintiles of DII score ${ }^{a}$

\begin{tabular}{|c|c|c|c|c|}
\hline \multirow[b]{3}{*}{ Median DII } & \multicolumn{3}{|c|}{ Quintiles of DII score } & \multirow[b]{3}{*}{$P$-trend } \\
\hline & $\overline{Q_{1}}$ & $\mathrm{Q}_{3}$ & $\mathrm{Q}_{5}$ & \\
\hline & -2.05 & -0.02 & +2.06 & \\
\hline \multicolumn{5}{|l|}{$\mathrm{IBS}-\mathrm{C}$} \\
\hline Crude & 1.00 & $0.84(0.55-1.26)$ & $1.12(0.76-1.64)$ & 0.24 \\
\hline Multivariable-adjusted $^{\text {b }}$ & 1.00 & $0.88(0.57-1.37)$ & $1.15(0.75-1.75)$ & 0.29 \\
\hline \multicolumn{5}{|l|}{ IBS-D } \\
\hline Crude & 1.00 & $0.90(0.53-1.50)$ & $1.33(0.83-2.13)$ & 0.45 \\
\hline Multivariable-adjusted $^{\mathrm{b}}$ & 1.00 & $0.95(0.54-1.66)$ & $1.37(0.81-2.30)$ & 0.49 \\
\hline \multicolumn{5}{|l|}{ IBS-M } \\
\hline Crude & 1.00 & $2.33(1.25-4.32)$ & $1.90(1.00-3.59)$ & 0.16 \\
\hline Multivariable-adjusted $^{\mathrm{b}}$ & 1.00 & $2.31(1.21-4.39)$ & $1.65(0.84-3.23)$ & 0.25 \\
\hline \multicolumn{5}{|l|}{ IBS-U } \\
\hline Crude & 1.00 & $1.02(0.65-1.61)$ & $1.19(0.76-1.85)$ & 0.96 \\
\hline Multivariable-adjusted $^{\mathrm{b}}$ & 1.00 & $1.06(0.65-1.72)$ & $1.21(0.75-1.94)$ & 0.92 \\
\hline
\end{tabular}

which was used in this study, might not reflect IBS severity. In addition, having low number of patients with severe IBS in the current study might provide another reason. Most patients in the study have indicated that they have mild to moderate abdominal pain. Further studies with prospective design are warranted to shed light on this issue.

There are several mechanisms through which a proinflammatory diet might influence IBS. Patients with IBS have higher levels of inflammatory cytokines. A proinflammatory diet can increase systemic inflammation [13]; therefore, it might be involved in the incidence and exacerbation of IBS symptoms. Consumption of proinflammatory diet has been linked with obesity [15]. Small bowel and colonic transit alteration in obese subjects might also explain IBS symptoms [41].

\section{Conclusion}

Our study has several strengths. This is the first study that examined the association of a pro-inflammatory diet and odds of IBS. Large sample size and taking the role of potential confounders into account are among other strengths of the present study. In addition, dietary habits which contribute to gastrointestinal disorders were considered as covariates in our analyses. Some limitations should also be considered when interpreting our findings. First, due to cross-sectional nature of the present study, causal relationships between DII score and IBS cannot be established. Therefore, further studies especially with prospective design are warranted to confirm our findings. Second, although we controlled for several potential confounders, the possibility of residual confounding cannot be excluded. In the current study, we used a validated DS-FFQ for dietary assessment and DII score calculation; however, measurement errors and misclassification of study participants cannot be avoided. In addition, some parameters were missing for DII score calculation due to lack of data on some antiinflammatory parameters including alcohol, eugenol, garlic, ginger, n- 3 fatty acids, n- 6 fatty acids, saffron, turmeric, flavan-3-ol, flavones, flavonols, flavonones, anthocyanidins, isoflavones, thyme/oregano and rosemary; therefore, we did not consider these dietary parameters in the calculation of DII score. The DII used in this study was based on earlier studies and it was not validated in Iranian population to examine whether it can really predict inflammation. However, based on the application of this score for prediction of different inflammatory-related conditions in the country, it seems that this index is a valid tool. For identification of IBS, we used questionnaire-based data. Although the validity of Rome III questionnaire has been shown in Iranian adults, the possibility of misclassification in terms of having IBS cannot be avoided.

In conclusion, we found that consumption of a proinflammatory diet was associated with increased odds of IBS. A significant association between DII score and IBS was observed in women, but not in men. In 
addition, a significant positive association was seen between DII score and IBS in overweight and obese subjects, but not in normal-weight participants. Further studies, especially with prospective design, are required to confirm our results.

\section{Abbreviations}

BMI: Body Mass Index; Cl: Confidence Interval; DII: Dietary Inflammatory Index; DS-FFQ: Dish-based Semi-quantitative Food Frequency Questionnaire; FDIl: Food-based Dietary Inflammatory Index; FGIDs: Functional Gastrointestinal Disorders; GPPAQ: General Practice Physical Activity Questionnaire; IBS: Irritable Bowel Syndrome; OR: Odds Ratio; SEPAHAN: Study on the Epidemiology of Psychological, Alimentary Health and Nutrition; WC: Waist Circumference

\section{Acknowledgements}

This study was extracted from a PhD dissertation that was approved by School of Nutritional Sciences and Dietetics, Tehran University of Medical Sciences (code 9321324004). The authors are thankful to participants of SEPAHAN project and authorities of Isfahan University of Medical Sciences for their excellent cooperation. We also appreciate the research council of Food Security Research Center for founding this project.

\section{Disclosure statement}

None of the authors had any personal or financial conflicts of interest.

\section{Authors' contributions}

ASM contributed to the search, statistical analyses, data interpretation and manuscript drafting. AHK and PA contributed to the conception, design and manuscript drafting. AE contributed to the conception, design, statistical analyses, data interpretation and manuscript drafting. AE supervised the study. All authors approved the final manuscript for submission.

\section{Funding}

This study was financially supported by Tehran University of Medical Sciences.

\section{Availability of data and materials}

The dataset used and analyzed during the current study is available from the corresponding author on a reasonable request.

\section{Ethics approval and consent to participate}

The study protocol was ethically approved by the Regional Bioethics Committee of Isfahan University of Medical Sciences. All participants provided written inform consent forms.

\section{Consent for publication}

All authors of this manuscript declared their consent for publication.

\section{Competing interests}

The authors declare that they have no competing interests.

\section{Author details}

'Students' Scientific Research Center, Tehran University of Medical Sciences, Tehran, Iran. ${ }^{2}$ Department of Community Nutrition, School of Nutritional Sciences and Dietetics, Tehran University of Medical Sciences, P.O. Box 14155-6117, Tehran, Iran. 'Department of Medicine, University of Alberta, Edmonton, Alberta, Canada. ${ }^{4}$ Integrative Functional Gastroenterology Research Center, Isfahan University of Medical Sciences, Isfahan, Iran. ${ }^{5}$ Obesity and Eating Habits Research Center, Endocrinology and Metabolism Molecular-Cellular Sciences Institute, Tehran University of Medical Sciences, Tehran, Iran. ${ }^{6}$ Department of Community Nutrition, School of Nutrition and Food Science, Isfahan University of Medical Sciences, Isfahan, Iran.

Received: 9 May 2019 Accepted: 24 September 2019 Published online: 11 November 2019

\section{References}

1. Khan S, Chang L. Diagnosis and management of IBS. Nat Rev Gastroenterol Hepatol. 2010;7(10):565-81.
2. Gunnarsson J, Simrén M. Efficient diagnosis of suspected functional bowel disorders. Nat Clin Pract Gastroenterol Hepatol. 2008;5(9):498-507.

3. Lovell RM, Ford AC. Global prevalence of and risk factors for irritable bowel syndrome: a meta-analysis. Clin Gastroenterol Hepatol. 2012;10(7):712-21.

4. Jahangiri P, Jazi MS, Keshteli AH, Sadeghpour S, Amini E, Adibi P. Irritable Bowel Syndrome in Iran: SEPAHAN Systematic Review No. 1. Int J Prev Med. 2012;3(Suppl 1):S1-9.

5. Matricon J, Meleine M, Gelot A, Piche T, Dapoigny M, Muller E, et al. Review article: Associations between immune activation, intestinal permeability and the irritable bowel syndrome. Aliment Pharmacol Ther. 2012;36(11-12): 1009-31.

6. Canavan C, West J, Card T. The epidemiology of irritable bowel syndrome. Clin Epidemiol. 2014;6:71-80

7. Philpott $H$, Gibson P, Thien F. Irritable bowel syndrome-An inflammatory disease involving mast cells. Asia Pac Allergy. 2011;1(1):36-42.

8. Hayes PA, Fraher MH, Quigley EM. Irritable bowel syndrome: the role of food in pathogenesis and management. Gastroenterol Hepatol (N Y). 2014; 10(3):164-74.

9. Akiho $\mathrm{H}$, Ihara $\mathrm{E}$, Nakamura K. Low-grade inflammation plays a pivotal role in gastrointestinal dysfunction in irritable bowel syndrome. World J Gastrointest Pathophysiol. 2010;1(3):97-105.

10. Sinagra E, Pompei G, Tomasello G, Cappello F, Morreale GC, Amvrosiadis G, et al. Inflammation in irritable bowel syndrome: Myth or new treatment target? World J Gastroenterol. 2016;22(7):2242-55.

11. El-Salhy M, Gundersen D. Diet in irritable bowel syndrome. Nutr J. 2015;14:36.

12. Shivappa N, Steck SE, Hurley TG, Hussey JR, Hebert JR. Designing and developing a literature-derived, population-based dietary inflammatory index. Public Health Nutr. 2014;17(8):1689-96.

13. Na W, Kim M, Sohn C. Dietary inflammatory index and its relationship with high-sensitivity C-reactive protein in Korean: data from the health examinee cohort. J Clin Biochem Nutr. 2018;62(1):83-8.

14. Shivappa N, Hebert JR, Marcos A, Diaz LE, Gomez S, Nova E, et al. Association between dietary inflammatory index and inflammatory markers in the HELENA study. Mol Nutr Food Res. 2017:61(6).

15. Ramallal R, Toledo E, Martinez JA, Shivappa N, Hebert JR, Martinez-Gonzalez MA, et al. Inflammatory potential of diet, weight gain, and incidence of overweight/ obesity: The SUN cohort. Obesity (Silver Spring). 2017;25(6):997-1005.

16. Neufcourt L, Assmann KE, Fezeu LK, Touvier M, Graffouillere L, Shivappa N, et al. Prospective association between the dietary inflammatory index and metabolic syndrome: findings from the SU.VI.MAX study. Nutr Metab Cardiovasc Dis. 2015;25(11):988-96.

17. Tyrovolas S, Koyanagi A, Kotsakis GA, Panagiotakos D, Shivappa N, Wirth $M D$, et al. Dietary inflammatory potential is linked to cardiovascular disease risk burden in the US adult population. Int J Cardiol. 2017;240:409-13.

18. Shivappa N, Hebert JR, Rashvand S, Rashidkhani B, Hekmatdoost A. Inflammatory Potential of Diet and Risk of Ulcerative Colitis in a CaseControl Study from Iran. Nutr Cancer. 2016;68(3):404-9.

19. Buscail C, Sabate JM, Bouchoucha M. Western Dietary Pattern Is Associated with Irritable Bowel Syndrome in the French NutriNet Cohort. Nutrients. 2017;9(9)

20. Khayyatzadeh SS, Esmaillzadeh A, Saneei P, Keshteli AH, Adibi P. Dietary patterns and prevalence of irritable bowel syndrome in Iranian adults. Neurogastroenterol Motil. 2016:28(12):1921-33.

21. Salari-Moghaddam A, Keshteli AH, Esmaillzadeh A, Adibi P. Empirically derived food-based inflammatory potential of the diet, irritable bowel syndrome, and its severity. Nutrition. 2019;63-64:141-7.

22. Adibi P, Keshteli AH, Esmaillzadeh A, Afshar H, Roohafza H, BagherianSararoudi R, et al. The study on the epidemiology of psychological, alimentary health and nutrition (SEPAHAN): overview of methodology. J Res Med Sci. 2012;17.

23. Keshteli AH, Esmaillzadeh A, Rajaie S, Askari G, Feinle-Bisset C, Adibi P. A dish-based semi-quantitative food frequency questionnaire for assessment of dietary intakes in epidemiologic studies in Iran: design and development. Int J Prev Med. 2014:5(1):29.

24. Shivappa N, Steck SE, Hurley TG, Hussey JR, Ma Y, Ockene IS, et al. A population-based dietary inflammatory index predicts levels of C-reactive protein in the Seasonal Variation of Blood Cholesterol Study (SEASONS). Public Health Nutr. 2014:17(8):1825-33.

25. Tabung FK, Steck SE, Zhang J, Ma Y, Liese AD, Agalliu I, et al. Construct validation of the dietary inflammatory index among postmenopausal women. Ann Epidemiol. 2015;25(6):398-405. 
26. Department of Health. The general practice physical activity questionnaire. London: Department of Health; 2009.

27. Oguma Y, Shinoda-Tagawa T. Physical activity decreases cardiovascular disease risk in women: review and meta-analysis. Am J Prev Med. 2004;26(5): 407-18.

28. Salari-Moghaddam A, Keshteli AH, Afshar H, Esmaillzadeh A, Adibi P. Association between dietary inflammatory index and psychological profile in adults. Clin Nutr. 2019;38(5):2360-8.

29. Hayes P, Corish C, O'Mahony E, Quigley EM. A dietary survey of patients with irritable bowel syndrome. J Hum Nutr Diet. 2014;27(Suppl 2):36-47.

30. Lovell RM, Ford AC. Effect of gender on prevalence of irritable bowel syndrome in the community: systematic review and meta-analysis. Am J Gastroenterol. 2012;107(7):991-1000.

31. Meleine M, Matricon J. Gender-related differences in irritable bowel syndrome: potential mechanisms of sex hormones. World J Gastroenterol. 2014;20(22):6725-43.

32. McDonough P, Walters V. Gender and health: reassessing patterns and explanations. Soc Sci Med. 2001;52(4):547-59.

33. Aro P, Ronkainen J, Talley NJ, Storskrubb T, Bolling-Sternevald E, Agréus L. Body mass index and chronic unexplained gastrointestinal symptoms: an adult endoscopic population based study. Gut. 2005;54(10):1377-83.

34. Svedberg P, Johansson S, Wallander MA, Hamelin B, Pedersen N. Extraintestinal manifestations associated with irritable bowel syndrome: a twin study. Aliment Pharmacol Ther. 2002;16(5):975-83.

35. Park HS, Park JY, Yu R. Relationship of obesity and visceral adiposity with serum concentrations of CRP, TNF-alpha and IL-6. Diabetes Res Clin Pract. 2005;69(1):29-35.

36. Undseth R, Berstad A, Valeur J. Systemic symptoms in irritable bowel syndrome: an investigative study on the role of enterocyte disintegrity, endotoxemia and inflammation. Mol Med Rep. 2016;14(6):5072-6.

37. Fysekidis M, Bouchoucha M, Bihan H, Reach G, Benamouzig R, Catheline J$M$. Prevalence and co-occurrence of upper and lower functional gastrointestinal symptoms in patients eligible for bariatric surgery. Obes Surg. 2012;22(3):403-10.

38. Sadik R, Björnsson E, Simrén M. The relationship between symptoms, body mass index, gastrointestinal transit and stool frequency in patients with irritable bowel syndrome. Eur J Gastroenterol Hepatol. 2010;22(1):102-8.

39. Talley NJ, Howell S, Poulton R. Obesity and chronic gastrointestinal tract symptoms in young adults: a birth cohort study. Am J Gastroenterol. 2004; 99(9):1807-14

40. Delgado-Aros S, Locke GR 3rd, Camilleri M, Talley NJ, Fett S, Zinsmeister AR, et al. Obesity is associated with increased risk of gastrointestinal symptoms: a population-based study. Am J Gastroenterol. 2004;99(9):1801-6.

41. Pickett-Blakely O. Obesity and irritable bowel syndrome: a comprehensive review. Gastroenterol Hepatol (N Y). 2014;10(7):411-6.

\section{Publisher's Note}

Springer Nature remains neutral with regard to jurisdictional claims in published maps and institutional affiliations.

Ready to submit your research? Choose BMC and benefit from:

- fast, convenient online submission

- thorough peer review by experienced researchers in your field

- rapid publication on acceptance

- support for research data, including large and complex data types

- gold Open Access which fosters wider collaboration and increased citations

- maximum visibility for your research: over $100 \mathrm{M}$ website views per year

At $\mathrm{BMC}$, research is always in progress.

Learn more biomedcentral.com/submissions 